\title{
Chromatographic Separations and Sample Extractions for Polycyclic Aromatic Hydrocarbons
}

\author{
Walter B. Wilson ${ }^{1}$
}

Published online: 13 February 2020

(c) This is a U.S. government work and its text is not subject to copyright protection in the United States; however, its text may be subject to foreign copyright protection 2020

Polycyclic aromatic hydrocarbons (PAHs) represent a diverse class of environmentally important chemical pollutants with potential mutagenic and carcinogenic properties. PAHs are highly researched in the literature with almost 60,000 publications through Web of Science dating back to 1911. PAHs have been identified in a wide range of environmental and combustion-related samples such as water, sediments, air particulate, coal tar, crude oil, and diesel particulate samples. One of the greatest challenges in the determination of PAHs in these samples are the complexity of the natural-matrix material and the multitude of isomeric structures.

Since the late 1970s, PAHs are typically quantified in these samples by a single chromatographic technique such as reversed-phase liquid chromatography (RPLC) with absorbance or fluorescence detection and gas chromatography coupled to mass spectrometry (GC-MS). In addition, sample extraction and clean-up methods have continuously evolved for PAH analysis to include normal-phase LC, liquid-liquid extraction (LLE), Soxhlet extraction, supercritical fluid extraction, pressurized fluid extraction, microwave-assisted extraction, solid-phase extraction, and solid-phase microextraction (SPME). In the past decade, improvements in PAH analysis have focused on developing highly efficient, selective, sensitive, reproducible, and green analytical methods.

The seven original papers presented in this virtual issue highlight some of the diverse work published in CHROMATOGRAPHIA over the last 2 calendar years for PAH research [1-7]. These publications exemplify the range of analytical techniques applicable to PAH separations and sample extraction, via dealing with RPLC [1], GC-MS [2-5], LLE

Walter B. Wilson

walter.wilson@nist.gov

1 Chemical Sciences Division, Materials Measurements Laboratory, National Institute of Standards and Technology, Gaithersburg, MD 20899, USA
[5], and SPME [6, 7]. The highlighted papers emphasize the synthesis and/or understanding of the retention mechanisms involved with RPLC [1], GC [2-4], and SPME [6, 7]. PAHs were measured in natural-matrix samples such as diesel particulate extract [1], soil [5], and real-world water samples [6, 7].

The papers included in the Virtual Issue on Chromatographic Separations and Sample Extractions for Polycyclic Aromatic Hydrocarbons will be accessible for free until the end of this month. We encourage all our readers to take a look at what the journal has published in this area. We expect contributions to the journal in these areas to continue to grow and welcome your submissions.

\section{References}

1. Wilson WB, Wise SA, Sander LC (2019) Development of a reversed-phase liquid chromatography and fluorescence method with multichannel selective wavelength detection for the determination of benzo[a]pyrene and six of its isomers. Chromatographia 82:499-508. https://doi.org/10.1007/s10337-018-3621-2

2. Oña-Ruales JO, Ruiz-Morales Y, Alvarez-Ramirez F, Wilson WB, Wise SA (2018) The influence of aromaticity in gas chromatography retention: the case of polycyclic aromatic sulfur heterocycles. Chromatographia 81:1219-1229. https://doi.org/10.1007/s1033 7-017-3465-1

3. Wang B, Liu J, Li X, Wu B (2018) Synthesis and applications of a novel 3,4-Bis(2-fluoro-5-trifluoromethyl phenyl)-2,5-diphenyl phenyl grafted polysiloxane stationary phase. Chromatographia 81:1219-1229. https://doi.org/10.1007/s10337-018-3556-7

4. Liu J, Xu L, Bai J, Wu B (2019) Ethyl carbazole-grafted polysiloxane as stationary phase for gas chromatography. Chromatographia 82:671-682. https://doi.org/10.1007/s10337-019-03694-0

5. Yazdanfar N, Shamsipur M, Ghambarian M, Esrafili A (2018) A highly sensitive dispersive microextraction method with magnetic carbon nanocomposites coupled with dispersive liquid-liquid microextraction and two miscible stripping solvents followed by GC-MS for quantification of 16 PAHs in environmental samples. Chromatographia 81:487-499. https://doi.org/10.1007/s1033 7-018-3469-5 
6. Feng J, Sun M, Wang X, Tian Y, Luo C, Feng J (2018) Barium sulfate nanoparticles as a coating for solid-phase microextraction of polycyclic aromatic hydrocarbons in aqueous samples. Chromatographia 81:1287-1292. https://doi.org/10.1007/s1033 7-018-3568-3

7. Tian Y, Sun M, Wang X, Luo C, Feng J (2018) A nanospherical metal-organic framework UiO-66 for solid-phase microextraction of polycyclic aromatic hydrocarbons. Chromatographia 81:10531061. https://doi.org/10.1007/s10337-018-3524-2

Publisher's Note Springer Nature remains neutral with regard to jurisdictional claims in published maps and institutional affiliations.

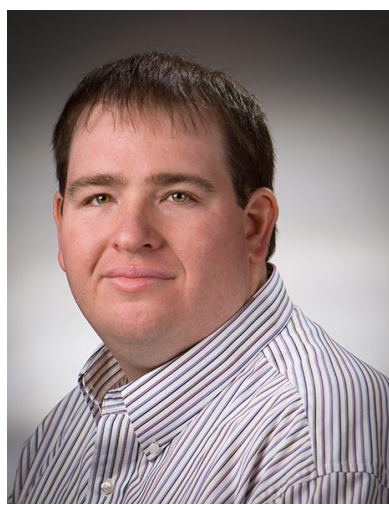

Walter Brent Wilson received his Ph. D. in Analytical Chemistry from the University of Central Florida (UCF) in August 2014, under the direction of Andres D. Campiglia. His research involved the development of new methods for the extraction, separation, and spectroscopic detection of polycyclic aromatic compounds (PACs) in environmental samples. In April 2015, he was awarded the Outstanding Dissertation Award from the College of
Sciences at UCF. Prior to attending UCF, he received a Bachelor of Science degree at Greensboro College and a Master of Science degree at the University of North Carolina of Greensboro. In February 2015, he was awarded a 2-year National Research Council post-doctoral fellowship at the National Institute of Standards and Technology (NIST), Chemical Sciences Division, under the direction of Stephen A. Wise and Lane C. Sander. His research focused on shape-selective separations of PACs using normal-phase and reversed-phase liquid chromatography. After completion of this fellowship, he joined the Organic Chemical Measurement Science Group at NIST as a research chemist. $\mathrm{He}$ is currently leading the efforts at NIST on developing a Cannabis Quality Assurance Program and Reference Materials (RMs). He is working to develop new chromatographic and spectroscopic methods for measuring multiple cannabinoids in Cannabis products of different sample matrix with specific interest but not limited to measuring $\Delta^{9}$-THC, THC, CBD, and CBDA. In addition, his research focuses on the certification of tobacco and dietary supplement natural product RMs for a variety of chemical species and contaminants. He has published 36 publications in international peer-reviewed journals and has had over 50 presentations at multiple local, national, and international conferences. Since 2018, he has been the vice president/program chair for the Washington Chromatography Discussion Group. 